\title{
Rapid fabrication of microrings with complex cross section using annular vortex beams
}

\author{
Chenchu Zhang (张晨初) $)^{1^{*}}$, Hanchang Ye (叶罕昌) $)^{1}$, Rui Cao (曹 锐) ${ }^{2}$, Shengyun Ji (姬生云) ${ }^{3}$, Heng Zhang (张 衡) ${ }^{1}$, \\ Linhan Zhao (赵麟寒) ${ }^{1}$, Sizhu Wu (吴思竹) ${ }^{1 * *}$, and Hua Zhai (翟 华) \\ ${ }^{1}$ Anhui Province Key Laboratory of Aerospace Structural Parts Forming Technology and Equipment, Institute of Industry and Equipment Technology, Hefei \\ University of Technology, Hefei 230009, China \\ ${ }^{2}$ Caltech Optical Imaging Laboratory, Andrew and Peggy Cherng Department of Medical Engineering, California Institute of Technology, Pasadena, \\ CA 91125, USA \\ 3 Hefei National Laboratory for Physical Sciences at the Microscale and CAS Key Laboratory of Mechanical Behavior and Design of Materials, Department of \\ Precision Machinery and Precision Instrumentation, University of Science and Technology of China, Hefei 230026, China
}

*Corresponding author: hfutzcc@hfut.edu.cn

${ }^{*}$ Corresponding author: sizhuwu@hfut.edu.cn

Received July 28, 2021 | Accepted August 31, 2021 | Posted Online October 14, 2021

\begin{abstract}
A ring-shaped focus, such as a focused vortex beam, has played an important role in microfabrication and optical tweezers. The shape and diameter of the ring-shaped focus can be easily adjusted by the topological charge of the vortex. However, the flow energy is also related to the topological charge, making the individual control of diameter and flow energy of the vortex beam impossible. Meanwhile, the shape of the focus of the vortex beam remains in the hollow ring. Expanding the shape of focus of structural light broadens the applications of the vortex beam in the field of microfabrication. Here, we proposed a ring-shaped focus with controllable gaps by multiplexing the vortex beam and annular beam. The multiplexed beam has several advantages, such as the diameter and flow energy of the focal point can be individually controlled and are not affected by the zero-order beam, and the gap size and position are controllable.
\end{abstract}

Keywords: microfabrication; femtosecond laser; structural beams; microring.

DOI: 10.3788/COL202220.023801

\section{Introduction}

A ring-shaped focus with a uniform hollow light distribution has an important role in the fields of optical tweezers ${ }^{[1]}$, microfabrication $^{[2]}$, and communication ${ }^{[3]}$. In the area of two-photon polymerization (TPP) fabrication, applying the ring-shaped focus instead of the conventional single-point focus allows polymerizing a tubular structure in a single exposure, which greatly improves the fabrication efficiency compared to the point-to-point scanning strategy. Until now, some very specific distributions, including Bessel beams ${ }^{[4]}$, spatially shifted vortex beams ${ }^{[5]}$, double-helix beams ${ }^{[6]}$, and ring-shaped beams ${ }^{[7]}$, have been explored for parallelization of the direct laser writing or even single-shot microfabrication. Among these methods, Bessel beams ${ }^{[8]}$ and vortex beams ${ }^{[9,10]}$ have been widely applied to generate ring-shaped foci. With the flexible control of parameters of Bessel and vortex beams, the diameter of ring-shaped focus can be easily adjusted. By changing the polarization state of an incident light, switchable spatial arrangement of the donutshaped focusing beams through an engineered microsphere can be tuned ${ }^{[11]}$. More than that, triangle and rectangle hollow foci can be also achieved by combining the Bessel beam and vortex beam $^{[12]}$, and an ellipse donut focus can be generated by cross phase $^{[13]}$.

Researches have made tremendous achievements in multidimensional manipulation of wave fields based on artificial microstructures ${ }^{[14]}$. Compared with the microstructure-based manipulation approaches, a flexible and commonly used method to generate ring-shaped beams is to use a spatial light modulator (SLM) to load the phase of the Bessel or vortex beam. In the case of the vortex beam, the shape and flow energy of optical vortices are controllable by more parameters ${ }^{[15]}$, including topological charge $l$, fold number $m$, and modulation depth $a$, which means that the laser pattern can be more flexibly structured. The traditional ideal vortex beam is generated by directly applying the spiral phase distribution $\exp (i l \varphi)$ to the incident laser beam, which can theoretically convert almost $100 \%$ of the incident radiation into a spiral vortex beam. However, due to the limitation of topological charge $l$, the diameters of donut vortex beams can be controlled by the topological charge 
in a relatively small range. Firstly, the topological charge should be an integer to make sure the focus remains ring-shaped. Secondly, the diameter of ring-shaped focus has a positive relationship with the topological charge. However, due to the limitation of pixelate of the SLM, the spiral phase with too high topological charge would result in distortion. In order to broaden the intensity distribution and increase the flow energy of the vortex beam, some unconventional vortex beams with nonlinear spiral phase distribution ${ }^{[16]}$ or fractional topological charges $^{[17,18]}$ were introduced by applying nonlinear spiral phase distribution increased with the azimuthal angle or even with fractional topological charge $l$. Until now, the application of these unconventional vortex beams in the field of TPP fabrication has not been studied.

Here, we present a novel annular-vortex beam (AVB). The AVB is generated by adding the phase of vortex and annular Fresnel lens together. The AVB has the advantages of changeable diameter, high uniformity, and being not affected by the zerothorder beam (ZOB). The ZOB is an undesired intensity distribution in the center of focus, which mainly has two causes: the deadzone between active pixels and the unmodified reflected energy ${ }^{[19]}$. To avoid the $\mathrm{ZOB}$ influencing the fabrication, researches use diffraction gratings to shift the ring-shaped beam away from the center ${ }^{[20]}$. However, the shift results in lower energy utilization and further pulling down of the fabrication efficiency.

In this Letter, optical vortices are generated by imprinting a helical phase on an incident femtosecond laser beam using a liquid crystal phase only SLM. The focusing properties of optical vortices are investigated with the Debye vectoral diffraction theory ${ }^{[21]}$. The influence of AVB's topological charge $l$ and zeroth-order width on the intensity distribution has been studied theoretically and experimentally. Microrings with and without controllable gaps are fabricated by simply exposing the AVBs in the photoresist. Furthermore, the relationship between phase factors and microring parameters is quantitatively studied. The diameters and gap sizes of fabricated microrings are well controlled by modulating the phase factors of the AVBs. On this basis, microrings with controllable geometry parameters and even 3D spiral microtubes are easily achieved. We believe the proposed approach can find potential applications in fabrication of complex shape silica microlenses ${ }^{[22]}$ and flexibly configured waveguide geometries in optical crystals ${ }^{[23]}$.

\section{Experiments and Methods}

The generation principle of AVB is shown in Fig. 1. A ringshaped focus can be generated by a conventional vortex beam containing a chiral wavefront or an annular Fresnel lens with a plane wavefront. For the former one, the diameter of ringshaped focus can be adjusted by the topological charge of the vortex beam, as shown in Fig. 1(b). According to the simulation, the diameters of foci are almost linear to the topological charges. However, both the diameter and the azimuthal energy flow are related to the topological charge, making the diameter
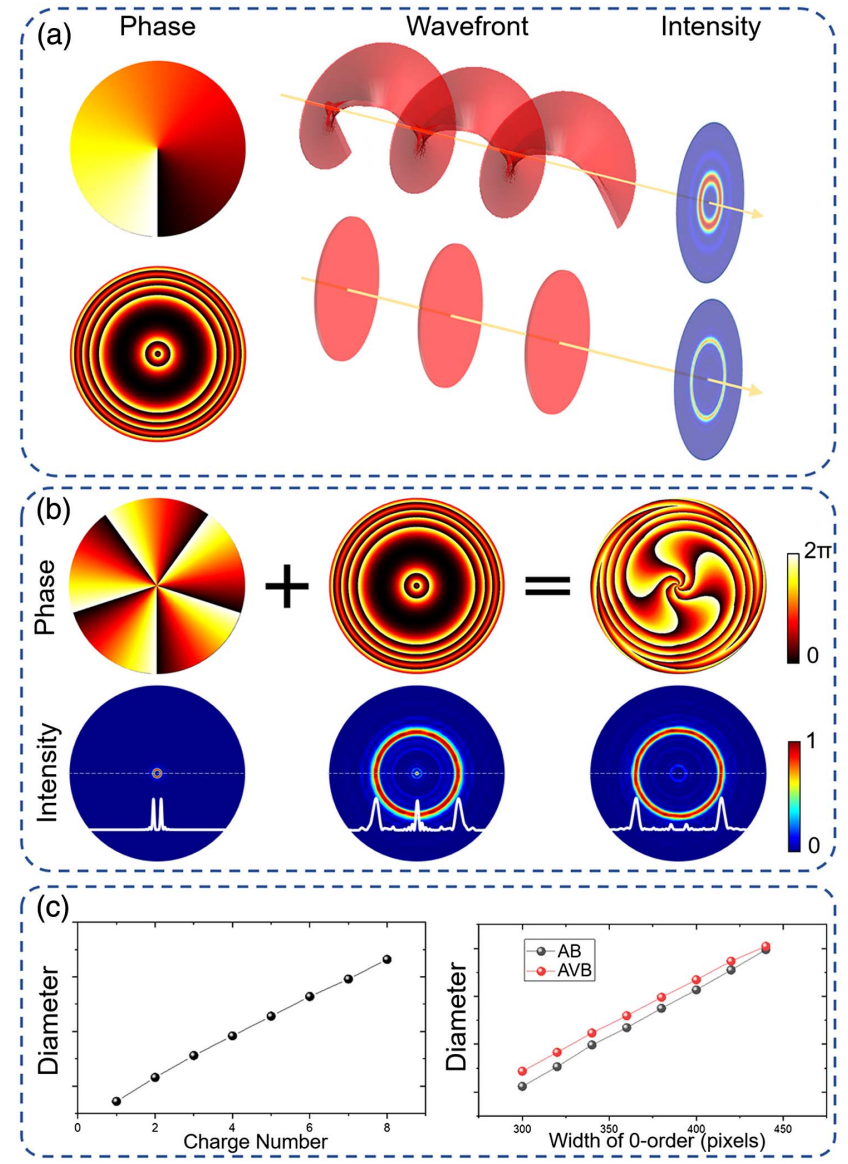

Fig. 1. (a) Generation schematic of AVB. (b) Simulated foci of vortex beam, $A B$, and AVB. (c) Diameter of ring-shaped focus versus the topological charge of vortex beams, and widths of zeroth-order AB and AVB.

unable to be individually changed without affecting the chiral characteristics.

On the other hand, the annular beam (AB) generated by the annular Fresnel lens does not contain a chiral wavefront. But, the diameter of the $\mathrm{AB}$ can be adjusted in a wider range compared to the vortex beam. Here, by multiplexing the vortex phase with an annular Fresnel lens, an AVB is presented, which contains chiral energy flow and wider diameter range. Meanwhile, the change of focus diameter does not relate to the topological charge of the vortex beam, which means the diameter of the ring-shaped focus and the chiral energy flow can be controlled individually. The unique property may find potential applications in the fields of optical tweezers and microfabrication. The generation of AVB is shown in Fig. 1(a), and the phase of the annular Fresnel lens is multiplexed to a vortex phase.

The mathematical expression of AVB can be written as follows:

$$
t(r, \theta)=\exp \left[\frac{i k\left(r-r_{0}\right)^{2}}{2 z}+i l \varphi(\theta)\right]
$$

where $k$ is the wave number, $r_{0}$ is the radius of the zeroth-order band of the annular Fresnel lens, $z$ is the focal length of the 
annular Fresnel lens, $l$ is the topological charge, and $\varphi(\theta)$ is the azimuthal angle around the optical axis.

As the modulation range of SLM used in the following experiment is from 0 to $2 \pi$, the multiplexed phase takes the remainder of $2 \pi$. The simulation of focus is carried out by the HuygensFresnel diffractive integral, which is shown as follows:

$$
\begin{aligned}
E(x, y, z)= & \frac{\exp (i k z)}{i \lambda z} \iint_{-\infty}^{\infty} E\left(x_{1}, y_{1}\right) \\
& \times \exp \left\{\frac{i k}{2 z}\left[\left(x-x_{1}\right)^{2}+\left(y-y_{1}\right)^{2}\right]\right\} \mathrm{d} x_{1} \mathrm{~d} y_{1},
\end{aligned}
$$

where $x, y$ are the coordinates for the initial field at the backside of the lens, while $x_{1}, y_{1}$ are the coordinates at the observation plane. $z$ is the distance from the lens back surface to the observation plane. $k$ is the wave number.

The corresponding simulated intensity distribution of the ring-shaped focus is shown at the bottom of Fig. 1(b). It can be clearly seen that there is almost no energy distribution in the center of the AVB, which means that the AVB does not suffer from the influence of the ZOB. The diameters of vortex beams, $\mathrm{ABs}$, and $\mathrm{AVBs}$ are almost also linear to the topological charges and the zeroth-order width of the annular Fresnel lens, respectively [Fig. 1(c)].

\section{Results}

To further analyze the principle of AVB, the intensity distribution has been investigated in both experiment and simulation. Figure 2(a) shows a schematic diagram of the experimental setup for femtosecond laser TPP fabrication.

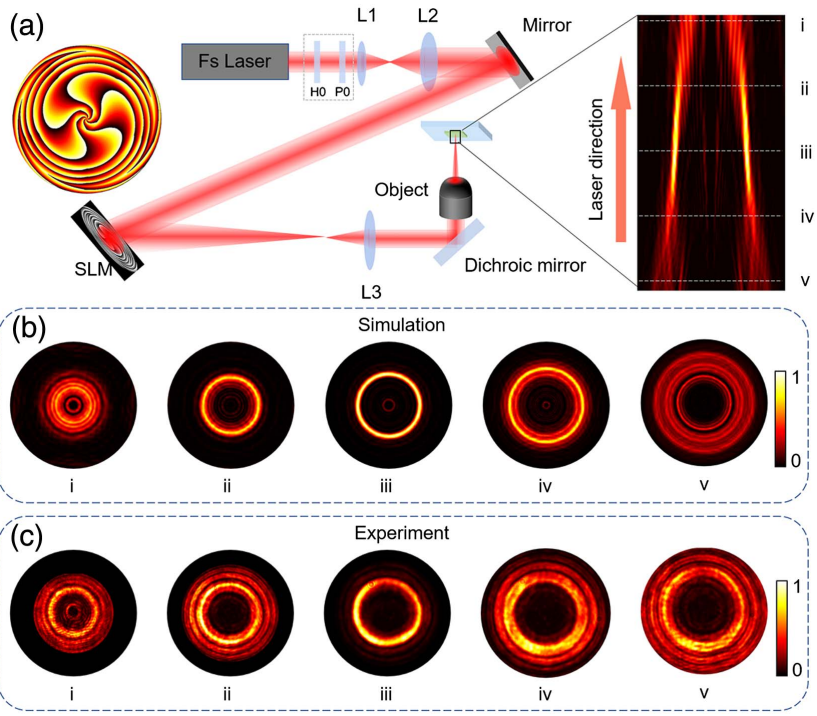

Fig. 2. (a) Experimental setup of AVB-based fabrication system. The right is the simulated intensity distribution along the propagation direction. (b) Simulated and (c) experimentally tested intensity distributions in different planes corresponding to dashed lines shown in (a).
The laser source is a mode-locked Ti:sapphire ultrafast oscillator (Coherent, Chamleon Vision-S) with the central wavelength of $800 \mathrm{~nm}$, pulse duration of $75 \mathrm{fs}$, and repetition rate of $80 \mathrm{MHz}$. After passing a beam collimation system with a $4 f$ configuration, which consisted of lens 1 and lens 2 , the beam is expanded to the size slightly larger than that of the SLM to ensure better modulation effects. The SLM loads computer generated holograms (CGHs), which are tailored to a circle to better match the incident light. It is worth noting that the CGHs do not need to be pretreated to shift the zero-order light away from the other modulated light. Then, the modulated beam is self-focused and contracted before being focused by the objective lens by a collimation lens. A $60 \times 1.35 \mathrm{NA}$ oil immersion objective lens was used to focus the phase modulated femtosecond laser beam into the photoresist sample. The intensity simulation is carried out by the Debye diffraction theory, as the NA of the objective is too high to satisfy the paraxial approximation condition. The simulated intensity distribution in the meridian plane along the propagation direction is shown in the right of Fig. 1(a). Along the direction of propagation, the diameter of the donut focus has a little shrink, which is mainly because the area of inner zones is smaller than that of outer zones. The intensity distribution in the sagittal plane has been tested both in simulation [Fig. 2(b)] and experiment [Fig. 2(c)]. The measured planes are taken in the focal plane (iii) and every $20 \mu \mathrm{m}$ away from the focal plane. From the simulated and experimental results, we find the measured results are consistent with the simulation.

$\mathrm{AVBs}$ are characterized by two parameters: the zero-order width and the topological charge. Both affect the diameter of the ring-shaped focus. To further investigate the optical principles of AVBs, a series of AVBs with different zero-order width has been studied. The diameter of the ring-shaped focus can be adjusted by changing the zero-order width when the charge number is fixed. Figure 3(a) shows the phase of AVBs with different diameters. The charge numbers of AVBs in Fig. 3(a) are fixed to five. The simulation results are shown in Fig. 3(b), proving that the diameters (which are $18.0 \mu \mathrm{m}, 15.3 \mu \mathrm{m}, 13.0 \mu \mathrm{m}$, $10.7 \mu \mathrm{m}$, and $8.4 \mu \mathrm{m}$, respectively) are related to the zero-order width of the $\mathrm{AVB}$, and the resolutions of the donut focus remain constant. Meanwhile, the ZOB does not appear in the center of the light field, which means it does not need to shift the ZOB away from the center of the pattern by a blazed grating. The measured light fields agree well with the simulation ones, as shown in Fig. 3(c). In the measured results, the diameters of foci are $25.4 \mu \mathrm{m}, 20.6 \mu \mathrm{m}, 16.6 \mu \mathrm{m}, 13.0 \mu \mathrm{m}$, and $9.9 \mu \mathrm{m}$, respectively. The top-right corner of the donut focus is relatively darker than other parts. This phenomenon is caused by some small deviations in the optical setup, such as the peak of the incident Gaussian beam not illuminating the exact center of the SLM, or the slight misalignment of the beam axis from the vertical axis of the photoresist surface.

The AVBs are further applied in rapidly fabricating tubular microstructures, as shown in Fig. 3(d). Microrings with different diameters of $22.1 \mu \mathrm{m}, 17.3 \mu \mathrm{m}, 14.1 \mu \mathrm{m}, 11.0 \mu \mathrm{m}$, and $8.5 \mu \mathrm{m}$ are fabricated by exposing the corresponding AVBs. The exposure time required to process the microstructure from the left to the 

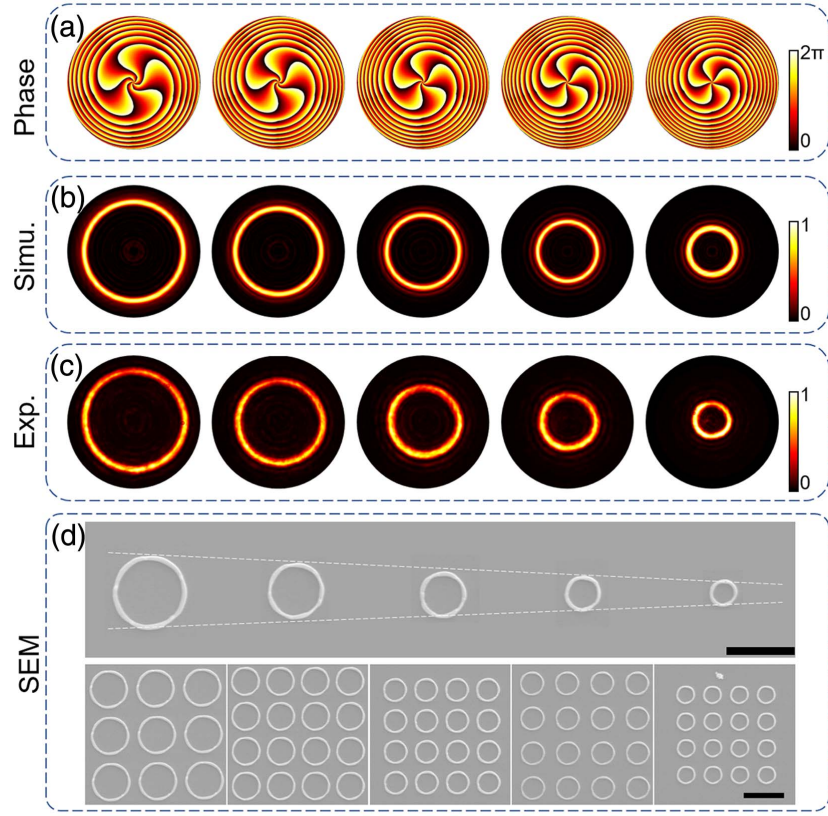

(e)
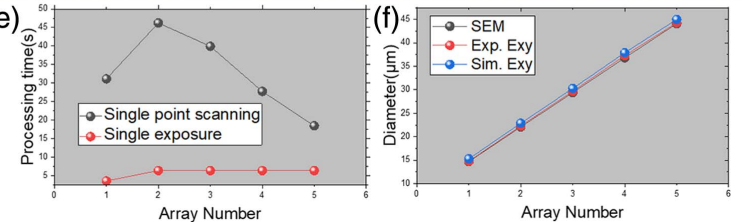

Fig. 3. (a) Phase of AVBs with different zero-order width. (b) Simulation intensity distribution of ring-shaped focus with different diameters. (c) Experimental intensity distribution of ring-shaped focus with different diameters. (d) SEM images of microtubes fabricated by single exposure of AVBs with different diameters. (e) Comparison of processing time between the single-exposure method and single-point scanning method. (f) Comparison of diameters of simulated ring-shaped foci, experimentally tested ring-shaped foci, and fabricated microstructures. Scale bars are $20 \mu \mathrm{m}$.

right in Fig. 3(d) is $500 \mathrm{~ms}, 400 \mathrm{~ms}, 300 \mathrm{~ms}, 200 \mathrm{~ms}$, and $100 \mathrm{~ms}$, when the incident laser power is maintained at $800 \mathrm{~mW}$. Microrings with larger diameters require more exposure time, as the energy density of those is weaker than that with smaller diameters. It can be seen the diameters of fabricated microstructures are linear to the zero-order width, as the simulations show before. The microring arrays are fabricated by exposing AVBs [Fig. 3(e)], showing that the consistency of microstructures keeps well. Benefiting from the high fabrication efficiency of AVBs, the processing time of a tube array is saved by a few times to even dozens of times, as shown in Fig. 3(e). More than that, the diameters of the simulation light field, experimentally tested light field, and fabricated microrings are almost the same, as shown in Fig. 3(f).

The AVB can not only carry the vortex phase with integer charge number, but can also carry that with the fractional charge number. By varying the topological charges, the optical fields with different gap sizes are realized. The generation principle is similar to that with an integer charge number, as shown in
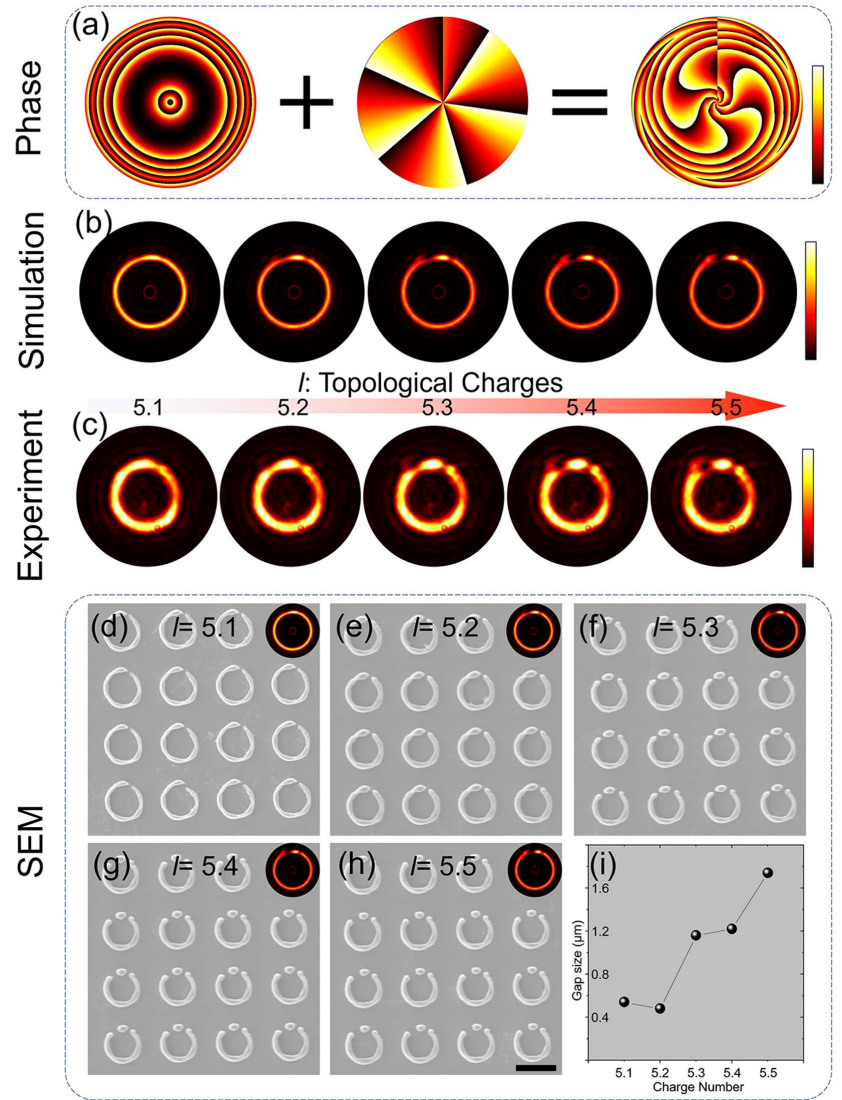

Fig. 4. (a) Generation principle of AVB with fractional topological charge. (b) Simulated gap-ring focus distribution. (c) Experimentally tested gap-ring focus distribution. The topological charges vary from 5.1 to 5.5 in (b) and (c). (d)-(h) SEM images of micro gap rings fabricated by single exposure of gapring focus. The top right corners are the corresponding foci. Scale bar is $10 \mu \mathrm{m}$. (i) Relationship between the gap size of fabricated microrings and the topological charges.

Fig. 4(a), and the phase of the annular Fresnel lens and the frictional topological charges are added, followed by a remainder of $2 \pi$.

The simulation of intensity distribution of the focus is shown in Fig. 4(b). The size of gaps increases with the topological charges increasing from 5.1 to 5.5 . The diameters of the gap rings are not obviously affected by the topological charge, as the topological charge only changes in a small range. The focus distribution is also tested in experiment, as shown in Fig. 4(c). The simulation results are in good agreement with the experimental ones.

\section{Conclusion}

In conclusion, we demonstrate a method for rapid fabrication of microrings with different diameters based on the AVBs. First, AVBs are simply realized by loading holograms with the combination of vortex beams and ABs. The diameter of AVBs can be flexibly controlled in a wide range with no $\mathrm{ZOB}$ in the center of 
the focus. Then, we are able to fabricate microrings with different diameters by a single exposure of focused AVBs. Finally, the AVBs with fractional topological charge have been generated. Since the topological charge AVB is not an integer, the intensity distribution of that is a hollow structure with controllable slits. The novel method developed in this work deepens the research into the flexible and fast TPP fabrication technique, and the fabricated microrings with slits are suitable for the number of important applications, such as artificial blood vessels and biomechanical research of blood cells.

\section{Acknowledgement}

This work was supported by the National Natural Science Foundation of China (Nos. 62005262 and 51875160) and Fundamental Research Funds for the Central Universities (Nos. PA2020GDSK0077 and PA2020GDKC0010).

\section{References}

1. Y. S. Liang, Y. A. Cai, Z. J. Wang, M. Lei, Z. L. Cao, Y. Wang, M. M. Li, S. H. Yan, P. R. Bianco, and B. L. Yao, "Aberration correction in holographic optical tweezers using a high-order optical vortex," Appl. Opt. 57, 3618 (2018).

2. J. Fischer and M. Wegener, "Three-dimensional optical laser lithography beyond the diffraction limit," Laser Photon. Rev. 7, 22 (2013).

3. Y.Xu, L. X. Guo, M. J. Cheng, and J. T. Li, "Controlling abruptly autofocusing vortex beams to mitigate crosstalk and vortex splitting in free-space optical communication," Opt. Express 26, 12605 (2018).

4. R. J. Winfield, B. Bhuian, S. O'Brien, and G. M. Crean, "Refractive femtosecond laser beam shaping for two-photon polymerization," Appl. Phys. Lett. 90, 111115 (2007).

5. H. Lin and M. Gu, "Creation of diffraction-limited non-Airy multifocal arrays using a spatially shifted vortex beam," Appl. Phys. Lett. 102, 084103 (2013).

6. S. Zhang, Y. Li, Z. Liu, J. Ren, Y. Xiao, H. Yang, and Q. Gong, "Two-photon polymerization of a three dimensional structure using beams with orbital angular momentum," Appl. Phys. Lett. 105, 061101 (2014).

7. C. Zhang, Y. Hu, J. Li, G. Li, J. Chu, and W. Huang, "A rapid two-photon fabrication of tube array using an annular Fresnel lens," Opt. Express 22, 3983 (2014).
8. S. Y. Ji, L. Yang, Y. L. Hu, J. C. Ni, W. Q. Du, J. W. Li, G. Zhao, D. Wu, and J. R. Chu, "Dimension-controllable microtube arrays by dynamic holographic processing as three-dimensional yeast culture scaffolds for asymmetrical growth regulation," Small 13, 701190 (2017).

9. K. K. Anoop, R. Fittipaldi, A. Rubano, X. Wang, D. Paparo, A. Vecchione, L. Marrucci, R. Bruzzese, and S. Amoruso, "Direct femtosecond laser ablation of copper with an optical vortex beam," J. Appl. Phys. 116, 113102 (2014).

10. L. Chen, R. K. Singh, A. Dogariu, Z. Chen, and J. X. Pu, "Estimating topological charge of propagating vortex from single-shot non-imaged speckle," Chin. Opt. Lett. 19, 022603 (2021).

11. Z. Yan and M. H. Hong, "Formation of polarization-dependent optical vortex beams via an engineered microsphere," Opt. Express 29, 11121 (2021).

12. Y. Liang, D. D. Qian, C. Xin, Z. J. Hu, S. Y. Ji, D. Wu, Y. L. Hu, J. W. Li, W. H. Huang, and J. R. Chu, "Direct laser writing of complex microtubes using femtosecond vortex beams," Appl. Phys. Lett 110, 221103 (2017).

13. C. Wang, Y. Ren, T. Liu, L. L. Chen, and S. Qiu, "New kind of HermiteGaussian-like optical vortex generated by cross phase," Chin. Opt. Lett. 18, 100501 (2020).

14. Y. B. Zhang, H. Liu, H. Cheng, J. G. Tian, and S. Q. Chen, "Multidimensional manipulation of wave fields based on artificial microstructures," OptoElectron. Adv. 3, 200002 (2020).

15. J. E. Curtis and D. G. Grier, "Structure of optical vortices," Phys. Rev. Lett. 90, 133901 (2003).

16. P. Li, S. Liu, T. Peng, G. Xie, X. Gan, and J. Zhao, "Spiral autofocusing Airy beams carrying power-exponent-phase vortices," Opt. Express 22, 7598 (2014).

17. M. Luo and Z. Wang, "Fractional vortex ultrashort pulsed beams with modulating vortex strength," Opt. Express 27, 36259 (2019).

18. J. Li, H. G. Liu, Y. Li, X. P. Wang, M. H. Sang, and X. F. Chen, "Directly generating vortex beams in the second harmonic by a spirally structured fundamental wave," Chin. Opt. Lett. 19, 060005 (2021).

19. O. Hernandez, M. Guillon, E. Papagiakoumou, and V. Emiliani, "Zero-order suppression for two-photon holographic excitation," Opt. Lett. 39, 5953 (2014).

20. C. W. Wang, Y. L. Hu, L. Yang, S. L. Rao, Y. L. Wang, D. Pan, S. Y. Ji, C. C. Zhang, Y. H. Su, W. L. Zhu, J. W. Li, D. Wu, and J. R. Chu, "Femtosecond Mathieu beams for rapid controllable fabrication of complex microcages and application in trapping microobjects," ACS Nano 13, 4667 (2019).

21. M. Gu, Advanced Optical Imaging Theory (Springer, 2000).

22. J. G. Hua, H. Ren, A. Jia, Z. N. Tian, L. Wang, S. Juodkazis, Q. D. Chen, and H. B. Sun, "Convex silica microlens arrays via femtosecond laser writing," Opt. Lett. 45, 636 (2020).

23. Y. C. Jia, S. X. Wang, and F. Chen, "Femtosecond laser direct writing of flexibly configured waveguide geometries in optical crystals: fabrication and application," Opto-Electron. Adv. 3, 190042 (2020). 